\title{
Solution Theory of Ginzburg-Landau Theory on BCS-BEC Crossover
}

\author{
Shuhong Chen ${ }^{1}$ and Zhong Tan ${ }^{2}$ \\ ${ }^{1}$ School of Mathematics and Statistics, Minnan Normal University, Zhangzhou, Fujian 363000, China \\ ${ }^{2}$ School of Mathematical Science, Xiamen University, Xiamen, Fujian 361005, China
}

Correspondence should be addressed to Shuhong Chen; shiny0320@163.com

Received 28 June 2014; Accepted 22 September 2014; Published 2 November 2014

Academic Editor: Salvador E. Venegas-Andraca

Copyright (C) 2014 S. Chen and Z. Tan. This is an open access article distributed under the Creative Commons Attribution License, which permits unrestricted use, distribution, and reproduction in any medium, provided the original work is properly cited.

We establish strong solution theory of time-dependent Ginzburg-Landau (TDGL) systems on BCS-BEC crossover. By the properties of Besov, Sobolev spaces, and Fourier functions and the method of bootstrapping argument, we deduce that the global existence of strong solutions to time-dependent Ginzburg-Landau systems on BCS-BEC crossover in various spatial dimensions.

\section{Introduction}

In this paper, we consider strong solutions to time-dependent Ginzburg-Landau systems,

$$
\begin{aligned}
&-i d u_{t}=\left(-\frac{d g^{2}+1}{U}+a\right) u+g[a+d(2 v-2 \mu)] \varphi_{B} \\
&+\frac{c}{4 m} \Delta u+\frac{g}{4 m}(c-d) \Delta \varphi_{B} \\
&-b\left|u+g \varphi_{B}\right|^{2 \sigma}\left(u+g \varphi_{B}\right), \\
& i \varphi_{B t}=-\frac{g}{U} u+(2 v-2 \mu) \varphi_{B}-\frac{1}{4 m} \Delta \varphi_{B}, \\
& u(x, 0)= u_{0}(x), \quad \varphi_{B}(x, 0)=\varphi_{B 0}(x), \quad \text { in } x \in \Omega, \\
& u(x, t)=0, \quad \varphi_{B}(x, t)=0, \quad \text { on }[0, \infty) \times \partial \Omega,
\end{aligned}
$$

where the definition domain of Laplacian $\Delta$ is $H^{2}(\Omega) \cap H_{0}^{1}(\Omega)$, and $\Omega$ is a bounded domain in $R^{n}$ with Lipschitzian boundary. The parameters $a, b, c, d$ and $m, U, g, v, \mu$ are all coupling coefficients, $t \geq 0, \sigma>0$, and $d=d_{r}+i d_{i}$ is generally complex.

The main purpose of this paper is to establish strong solutions theory of (1)-(4). The systems are the general form of Ginzburg-Landau theory for superfluid atomic Fermi gases describing the BCS-BEC crossover near the Feshbach resonance from the Fermion-Boson model (double-channel model) [1]. The BCS-BEC crossover phenomena have been found as early as in $1992[2,3]$. Due to the strange feature taken on by the quantum phenomena, it attracts many scientists' attention and interest [4-6]. Though so many results have been found, there are a few works got by mathematical framework.

Even though the Ginzburg-Landau theory can capture almost every unique feature that the superfluid exhibited macroscopically $[7,8]$, this leads to the fact that it played an important role in the history of superfluid atomic Fermi gases research. In fact, the Ginzburg-Landau equation (single-channel model) has proved fruitful for illustration of the connection between infinite dimensional dynamics and finite dimensional dynamical systems [7-11]. Thus, MachidaKoyama constructed a time-dependent Ginzburg-Landau theory for BCS-BEC crossover from the Fermion-Boson model [1]. Then, Chen and Guo found weak solutions [12] and classical solutions [13] theories of the time-dependent Ginzburg-Landau theory. In present paper, we would further show the global strong solutions of initial value problem (1)(4) under some suitable conditions.

In the strong solution theory, the admissible parameter values of $a, c, d, m, U$ and the dimension $n$ are interrelated. This arbitrariness contrasts sharply with the theory of weak solutions [10]. Luckily, we can overcome this problem by Fourier transform and using the properties of Fourier functions. However, the higher the dimension is, the harder 
the problem is [14]. The difficulty lies in obtaining a priori estimate of higher derivatives of solutions. Furthermore, the approaches in both $[15,16]$ could not be applied to the current paper, since the crossover between the states BCS and BEC can bring some technical difficulties. In order to handle these difficulties, we have to use the properties of Besov and Sobolev spaces and the bootstrapping argument. But what make things worse is that a priori estimate even in the case of nonlinear wave [17-20] requires some restrictions on the nonlinear term, which cause the fact that one just can obtain the desired result in the case of $n \leq 11$. Thus, combining all techniques of Fourier transform, Besov and Sobolev spaces, and the energy method and bootstrapping argument, we overcome these difficulties and establish the strong solutions theory of (1)-(4) in various spatial dimensions.

Theorem 1. Let $\Omega$ be a bounded domain in $R^{n}$, with Lipschitzian boundary. Assume that $U, c, d_{i}, m, b$ are positive coefficients and $a, g, \mu, \nu$ are real numbers. For $\sigma>0$, assume that

$$
\frac{1}{2} \sigma n-1<0
$$

or let $q>2$, provided

$$
\begin{gathered}
\left|d_{r}\right| \leq \frac{2 d_{i} \sqrt{q-1}}{q-2}, \\
\sigma n<\frac{2|d|}{|d|-d_{i}} .
\end{gathered}
$$

Then the problem (1)-(4) with $C^{2}$ initial conditions has a unique global strong solution.

Theorem 2. Under the coefficients' conditions of Theorem 1 , for $\sigma \geq 1 / 2$ and $n<2+2 / \sigma$, the problem (1)-(4) has a unique global strong solution if the dispersive parameters lie in the region of the $\left(c d_{r} / 4 m|d|^{2}, b d_{r} /|d|^{2}\right)$-plane, bounded by hyperbolae, which satisfies

$$
\frac{\left|d_{r}(c-4 m b)\right|}{\left|d_{i}(c-2 \sqrt{m b c})\right|} \leq \frac{\sqrt{2 \sigma+1}}{\sigma} \text {. }
$$

Remark 3. The relations between physics and mathematics are closed and they can complement each other. The physical phenomenon can validate the mathematical results; on the other hand, the mathematical results would provide the theoretical support for physical phenomena. For example, Theorem 1 means that, under suitable conditions, the transformation process of the two kinds of particles in BCS-BEC crossover region exists a smooth state. Therefore, in practice, we can control whether the smooth state should take on by adjusting the related conditions. Theorem 2 shows that, under certain conditions, we can decide that which region should take on the smooth state by adjusting parameters.

\section{Local Existence of Strong Solutions}

In this section, we would establish local strong solutions to (1)-(4). At first, the initial boundary value problem (1)-(2) may be rewritten as follows:

$$
\begin{aligned}
&\left(u+g \varphi_{B}\right)_{t} \\
&=\left(\frac{i a}{d}-\frac{i}{d U}\right)\left(u+g \varphi_{B}\right)+\frac{i g}{d U} \varphi_{B}+\frac{i c}{4 m d} \Delta\left(u+g \varphi_{B}\right) \\
&-\frac{i b}{d}\left|u+g \varphi_{B}\right|^{2 \sigma}\left(u+g \varphi_{B}\right), \\
& \varphi_{B t}=\frac{i g}{U} u-i(2 v-2 \mu) \varphi_{B}+\frac{i}{4 m} \Delta \varphi_{B} .
\end{aligned}
$$

For $\vec{u}=\left(\begin{array}{c}u(x, t)+g \varphi_{B}(x, t) \\ \varphi_{B}(x, t)\end{array}\right)$ with $\vec{u}_{0}=\left(\begin{array}{c}u_{0}+g \varphi_{B 0} \\ \varphi_{B 0}\end{array}\right),(1)-(4)$ become

$$
\begin{gathered}
\vec{u}_{t}=\left(A_{1}+i A_{2}\right) \vec{u}+\left(A_{3}+i A_{4}\right) \Delta \vec{u}+J(\vec{u}), \\
\vec{u}(0)=\vec{u}_{0}, \quad \vec{u}(x, t)=0, \quad \text { on }[0, \infty) \times \partial \Omega,
\end{gathered}
$$

where

$$
\begin{gathered}
A_{1}=\left(\begin{array}{cc}
\frac{(a U-1) d_{i}}{|d|^{2} U} & \frac{d_{i}}{|d|^{2} U} \\
0 & 0
\end{array}\right), \\
A_{2}=\left(\begin{array}{cc}
\frac{(a U-1) d_{r}}{|d|^{2} U} & \frac{g d_{r}}{|d|^{2} U} \\
\frac{g^{2}}{U} & -2 v+2 \mu-\frac{g^{2}}{U}
\end{array}\right), \\
A_{3}=\left(\begin{array}{cc}
\frac{c d_{i}}{4 m|d|^{2}} & 0 \\
0 & 0
\end{array}\right), \quad A_{4}=\left(\begin{array}{cc}
\frac{c d_{r}}{4 m|d|^{2}} & 0 \\
0 & \frac{1}{4 m}
\end{array}\right), \\
J(\vec{u})=\left(\begin{array}{cc}
-\frac{i b}{d}\left|u+g \varphi_{B}\right|^{2 \sigma} & \left(u+g \varphi_{B}\right) \\
0 &
\end{array}\right) .
\end{gathered}
$$

Obviously, $A_{3}$ is a nonnegative defined matrix.

Consider the evolution of $\vec{u}=\vec{u}(t)$ in a Banach space $X$ to be governed by the abstract initial-value problem (10) with $\vec{u}(0)=\vec{u}_{0} \in X$ and the perturbation $J$ is often a nonlinear and noncontinuous map over $X$. Then, via the corresponding integral equation

$$
\vec{u}(t)=S(t) \vec{u}(t)+\int_{0}^{t} S\left(t-t^{\prime}\right) J\left(\vec{u}\left(t^{\prime}\right)\right) d t^{\prime},
$$

assume that $J$ is a locally Lipschitz continuous map from $X$ into itself; that is,

(i) $\|J(\vec{u})\|_{X} \leq C_{b d}\left(\|\vec{u}\|_{X}\right)$ for every $\vec{u} \in X$,

(ii) $\|J(\vec{u})-J(\vec{v})\|_{X} \leq C_{\text {Lip }}\left(\|\vec{u}\|_{X},\|\vec{v}\|_{X}\right)$ for every $\vec{u}, \vec{v} \in X$, 
where $C_{b c}(\cdot)$ and $C_{\text {Lip }}(\cdot)$ are nondecreasing functions. Then, employing the contraction mapping theorem, one can get the following basic result.

Lemma 4 (local existence theorem). For every $\rho>0$ there exists a time $T(\rho)>0$ such that, for every initial data $\vec{u}_{0} \in X$, with $\left\|\vec{u}_{0}\right\| \leq \rho$, there exists a unique $\vec{u} \in C([0, T], X)$ satisfying the mild formulation (12). Moreover, $\vec{u}$ is a locally Lipschitz continuous function of $\vec{u}_{0}$.

Definition 5. A function $\vec{u} \in C([0, T], X)$ satisfying the mild formulation (12) is called a mild solution for the initial value problem (10).

Definition 6. A function $\vec{u}$ satisfying (10) with $C^{2}$ in space and $C^{1}$ in time is called a strong solution for the initial value problem (10).

Lemma 7 ( $L^{\infty}$-local strong solutions). For every $\rho>0$ there exists a time $T(\rho)>0$ such that for every initial data $\vec{u}_{0} \in$ $L^{\infty}(\Omega)$ with $\left\|\vec{u}_{0}\right\|_{L^{\infty}} \leq \rho$ there exists a unique

$$
\begin{aligned}
\vec{u} \in & C\left([0, T], L^{\infty}(\Omega)\right) \\
& \cap C\left((0, T], C^{2}(\Omega)\right) \cap C^{1}((0, T], C(\Omega)),
\end{aligned}
$$

satisfying the initial value problem (10). Moreover, for every initial data $\vec{u}_{0} \in C^{2}(\Omega)$, one has $\vec{u} \in C\left([0, T], C^{2}(\Omega)\right) \cap$ $C^{1}([0, T], C(\Omega))$.

Proof. By Fourier transform and the properties of Fourier functions, the associated operator $S(t)$ of (12) acting on $\vec{u} \in X$ can be written as a convolution, $S(t) \vec{u}=N_{t} * \vec{u}$, with its Green function $N_{t}=\sum_{l \in R^{n}} f_{t}(x+l)$ given by

$$
\begin{aligned}
f_{t}(x)= & \frac{1}{\left[4 \pi\left(A_{3}+i A_{4}\right) t\right]^{n / 2}} \\
& \times \exp \left[-\frac{|x|^{2}}{4\left(A_{3}+i A_{4}\right) t}+\left(A_{1}+i A_{2}\right) t\right] .
\end{aligned}
$$

The integral equation (12) recast in terms of this Green function takes the form

$$
\vec{u}(t)=N_{t} * \vec{u}_{0}+\int_{0}^{t} N_{t-\tau} * J(\vec{u}(\tau)) d \tau .
$$

We first appeal to $X=L^{\infty}(\Omega)$. The perturbation $J$ as a map from $L^{\infty}(\Omega)$ into itself is clearly locally Lipschitz continuous. Lemma 4 yields a unique mild solution $\vec{u}=$ $\vec{u}(t) \in C\left([0, T], L^{\infty}(\Omega)\right)$ of $(12)$ over a time interval $[0, T]$ that depends only on the $L^{\infty}$ norm of $\vec{u}_{0}$. This solution is the limit of a sequence $\left\{\vec{u}^{(l)}\right\}$ of successive iterates of (15), say, that defined by

$$
\begin{gathered}
\vec{u}^{(0)}(t)=N_{t} * \vec{u}_{0}, \\
\vec{u}^{(l+1)}(t)=N_{t} * \vec{u}_{0}+\int_{0}^{t} N_{t-\tau} * J\left(\vec{u}^{(l)}(\tau)\right) d \tau,
\end{gathered}
$$

that converge in $C\left([0, T], L^{\infty}(\Omega)\right)$ for some $T$ chosen sufficiently small such that the sequence contracts.
In order to elevate these mild solutions to strong solutions, we use standard bootstrapping argument. First, evoking the regularity of $N_{t}$, the gradient of (15) yields

$$
\nabla \vec{u}=\nabla N_{t} * \vec{u}_{0}+\int_{0}^{t} \nabla N_{t-\tau} * J(\vec{u}(\tau)) d \tau,
$$

Then, use the $L^{1}$-estimate of $\nabla N_{t}$ with

$$
\begin{aligned}
\left\|\nabla N_{t}\right\|_{L^{1}} & \leq \sum_{l \in R^{n}} \int_{\Omega}\left|\nabla f_{t}(x+l)\right| d x \\
& =\int_{R^{n}}\left|\nabla f_{t}(x)\right| d x \leq C_{n}\left(A_{3}^{2}+A_{4}^{2}\right)^{n / 4} \frac{e^{\left(A_{1}+i A_{2}\right) t}}{\sqrt{t}},
\end{aligned}
$$

where $C_{n}$ is a constant depending only on the dimension $n$, applied to the successive iterates (16) to show that each $\nabla \vec{u}^{(l)}$ lies in $C((0, T], C(\Omega))$ and that the sequence converges. It follows that $\vec{u} \in C\left((0, T], C^{1}(\Omega)\right)$ with

$$
\begin{aligned}
\|\nabla \vec{u}\|_{L^{\infty}} \leq & \left\|\nabla N_{t}\right\|_{L^{1}}\left\|\vec{u}_{0}\right\|_{L^{\infty}}+\int_{0}^{t}\left\|\nabla N_{t-\tau}\right\|_{L^{1}}\|J(\vec{u}(\tau))\|_{L^{\infty}} d \tau \\
\leq & C_{n}\left(A_{3}^{2}+A_{4}^{2}\right)^{n / 4} \frac{e^{\left(A_{1}+i A_{2}\right) t}}{\sqrt{t}}\left\|\vec{u}_{0}\right\|_{L^{\infty}} \\
& +C_{n}\left(A_{3}^{2}+A_{4}^{2}\right)^{n / 4} \int_{0}^{t} \frac{e^{\left(A_{1}+i A_{2}\right)(t-\tau)}}{\sqrt{t-\tau}}\|J(\vec{u}(\tau))\|_{L^{\infty}} d \tau .
\end{aligned}
$$

Then, if it was the case that $\vec{u}_{0} \in C^{1}(\Omega)$, then the singularity in this estimate at $t=0$ disappears and one sees that $\vec{u} \in C\left([0, T], C^{1}(\Omega)\right)$ and that $\nabla \vec{u}$ is a solution of

$$
\nabla \vec{u}=N_{t} * \nabla \vec{u}_{0}+\int_{0}^{t} N_{t-\tau} *[D J(\vec{u}(\tau)) \nabla(\vec{u}(\tau))] d \tau,
$$

where $D J(\vec{u})$ represents the derivative of $J(\vec{u})$ with respect to $\vec{u}$, where in this case $D J(\vec{u}) \nabla \vec{u}=\left(\begin{array}{c}\nabla J_{1} \\ \nabla J_{2}\end{array}\right)$ is given explicitly by

$$
\begin{aligned}
& \nabla J_{1}=-\left(\frac{b d_{i}}{|d|^{2}}+i \frac{b d_{r}}{|d|^{2}}\right) \\
& \times {\left[(\sigma+1)\left|u+g \varphi_{B}\right|^{2 \sigma} \nabla\left(u+g \varphi_{B}\right)\right.} \\
&\left.+\sigma\left|u+g \varphi_{B}\right|^{2 \sigma-2}\left(u+g \varphi_{B}\right)^{2} \nabla\left(\overline{u+g \varphi_{B}}\right)\right], \\
& \nabla J_{2}=0 .
\end{aligned}
$$

A repetition of the above regularity argument starting from (20) shows that $\vec{u}$ is in $C\left((0, T], C^{2}(\Omega)\right)$. Moreover, because (8) and (9) relate the first time derivative to the second space derivative, the solution must also be in $C^{1}((0, T], C(\Omega))$ and is therefore a strong solution of systems (1)-(4) so long as it is a mild solution.

Lemma 8 (local $C^{k}$ solutions). Let $\sigma \geq(1 / 2) l$ for some positive integer $l$. Then for every $\rho<0$ there exists a time 
$T(\rho)>0$ such that for every initial data $\vec{u}_{0} \in L^{\infty}(\Omega)$ with $\left\|\vec{u}_{0}\right\|_{L^{\infty}} \leq \rho$ there exists a unique

$$
\begin{aligned}
\vec{u} \in & C\left([0, T], L^{\infty}(\Omega)\right) \\
& \cap C\left((0, T], C^{l+2}(\Omega)\right) \cap C^{1}\left((0, T], C^{l}(\Omega)\right),
\end{aligned}
$$

satisfying (1)-(4). Moreover, for every initial data $\vec{u}_{0} \in$ $C^{l+2}(\Omega)$, one has

$$
\vec{u} \in C\left([0, T], C^{l+2}(\Omega)\right) \cap C^{1}\left([0, T], C^{l}(\Omega)\right) .
$$

Proof. From (21), we observe that the lowest degree of homogeneity for the factors $\left(u+g \varphi_{B}\right)$ and $\left(\overline{u+g \varphi_{B}}\right)$ appearing in a term of the $(l+1) s t$ derivative of $\left|u+g \varphi_{B}\right|^{2 \sigma}\left(u+g \varphi_{B}\right)$ will be $2 \sigma-l$, and this can be controlled whenever $\sigma \geq(1 / 2) l$. In that case, the bootstrapping argument will gain additional $l$ spatial derivatives, showing that the solution is in $C\left((0, T], C^{l+2}(\Omega)\right)$. This is Lemma 8.

Refinements of the basic existence argument given above greatly enlarge the class of initial data that evolve into strong solutions for finite times.

Lemma 9 ( $L^{p}$-local mild solutions). If $p$ satisfies

$$
q \leq p, \quad \sigma n<p
$$

then for every $\rho>0$ there exists a time $T(\rho)>0$ such that for every initial data $\vec{u} \in L^{p}(\Omega)$ with $\left\|\vec{u}_{0}\right\| \leq \rho$ and $\|\vec{u}\|_{E^{p, r}} \equiv$ $\sup \left\{\|\vec{u}\|_{L^{r} / L^{q}}: t \in[0, T]\right\}$, with $1+1 / r=1 / p+1 / q, r \geq 1+2 \sigma$, and $\|\vec{u}\|_{L^{r} / L^{q}}=\|\vec{u}\|_{L^{r}} /\left\|N_{t}\right\|_{L^{q}}$, there exists a unique

$$
\vec{u} \in E^{p, r}([0, T], \Omega) \cap C\left([0, T], L^{p}(\Omega)\right),
$$

satisfying the mild formulation (15) of the initial value problem (1)-(4).

Proof. Noting that function (14) satisfies the $L^{1}$-estimate

$$
\begin{aligned}
\left\|N_{t}\right\|_{L^{1}} & \leq \sum_{l \in R^{n}} \int_{\Omega}\left|f_{t}(x+l)\right| d x \\
& =\int_{R^{n}}\left|f_{t}(x)\right| d x=\left(A_{3}^{2}+A_{4}^{2}\right)^{n / 4} e^{\left(A_{1}+i A_{2}\right) t} .
\end{aligned}
$$

Controlling the $L^{\infty}(\Omega)$ norm of $N_{t}$ follows from the bound

$$
\begin{aligned}
& \left|N_{t}(x)\right| \\
& \leq \sum_{l \in R^{n}}\left|f_{t}(x+l)\right| \\
& \leq \frac{\left(A_{3}^{2}+A_{4}^{2}\right)^{n / 4}}{\left[4 \pi\left(A_{3}^{2}+A_{4}^{2}\right) t\right]^{n / 2}} e^{\left(A_{1}+i A_{2}\right) t} \sum_{l \in R^{n}} \exp \left(-\frac{|x+l|^{2}}{4 t\left(A_{3}^{2}+A_{4}^{2}\right)}\right) \\
& \leq \frac{\left(A_{3}^{2}+A_{4}^{2}\right)^{n / 4}}{\left[4 \pi\left(A_{3}^{2}+A_{4}^{2}\right) t\right]^{n / 2}} e^{\left(A_{1}+i A_{2}\right) t}\left\{C_{1}+C_{2}\left[\left(A_{3}^{2}+A_{4}^{2}\right) t\right]^{n / 2}\right\},
\end{aligned}
$$

for some positive absolute constants $C_{1}$ and $C_{2}$. By an interpolation argument, the $L^{q}(\Omega),\left(1 / q+1 / q^{*}=1\right)$ norm of $N_{t}$ is estimated by

$$
\begin{aligned}
& \left\|N_{t}(x)\right\|_{L^{q}} \\
& \leq\left\|N_{t}(x)\right\|_{L^{\infty}}^{1 / q^{*}}\left\|N_{t}(x)\right\|_{L^{1}}^{1 / q} \\
& \leq \frac{\left(A_{3}^{2}+A_{4}^{2}\right)^{n / 4} e^{\left(A_{1}+i A_{2}\right) t}}{\left[4 \pi\left(A_{3}^{2}+A_{4}^{2}\right) t\right]^{n /\left(2 q^{*}\right)}}\left\{C_{1}+C_{2}\left[\left(A_{3}^{2}+A_{4}^{2}\right) t\right]^{n / 2}\right\}^{1 /\left(q^{*}\right)} .
\end{aligned}
$$

Thus, one sees that

$$
\left\|N_{t}(x)\right\|_{L^{q}}=O\left(\frac{1}{t^{n / 2 q^{*}}}\right), \quad \text { as } t \longrightarrow 0 .
$$

Then, a direct $L^{r}$ estimate of the mild formulation (15) for every $r$ satisfying

$$
p \leq r, \quad 2 \sigma+1 \leq r
$$

yields

$$
\begin{aligned}
\|\vec{u}(x, t)\|_{L^{r}} & =\left\|N_{t}\right\|_{L^{q}}\left\|\vec{u}_{0}\right\|_{L^{p}}+\int_{0}^{t}\left\|N_{t-\tau}\right\|_{L^{2}}\|J(\vec{u}(x, \tau))\|_{L^{r}} d \tau \\
& \leq\left\|N_{t}\right\|_{L^{q}}\left\|\vec{u}_{0}\right\|_{L^{p}}+\int_{0}^{t}\left\|N_{t-\tau}\right\|_{L^{L^{\prime}}}\|\vec{u}(x, \tau)\|_{L^{r}}^{2 \sigma+1} d \tau,
\end{aligned}
$$

where $q$ and $s$ satisfy

$$
1+\frac{1}{r}=\frac{1}{q}+\frac{1}{p}, \quad 1+\frac{1}{r}=\frac{1}{s}+\frac{2 \sigma+1}{r} .
$$

The idea is to recast (32) as an inequality for the ratio

$$
\|\vec{u}(x, t)\|_{L^{r} / L^{q}} \equiv \frac{\|\vec{u}(x, t)\|_{L^{r}}}{\left\|N_{t}\right\|_{L^{q}}},
$$

whence obtaining

$$
\begin{aligned}
& \|\vec{u}(x, t)\|_{L^{r} / L^{q}} \\
& \leq\left\|\vec{u}_{0}\right\|_{L^{p}}+\frac{1}{\left\|N_{t}\right\|_{L^{q}}} \int_{0}^{t}\left\|N_{t-\tau}\right\|_{L^{L}}\left\|N_{\tau}\right\|_{L^{q}}^{2 \sigma+1}\|\vec{u}(x, \tau)\|_{L^{\prime} / L^{q}}^{2 \sigma+1} d \tau .
\end{aligned}
$$

It is easily seen from (30) that whenever

$$
\left(\frac{n}{2 q^{*}}\right)(2 \sigma+1)<1, \quad \frac{n}{2 s^{*}}+\left(\frac{n}{q^{*}}\right) \sigma<1,
$$

the condition

$$
\frac{1}{\left\|N_{t}\right\|_{L^{q}}} \int_{0}^{t}\left\|N_{t-\tau}\right\|_{L^{s}}\left\|N_{\tau}\right\|_{L^{q}}^{2 \sigma+1} d \tau \longrightarrow 0 \quad \text { as } \tau \longrightarrow 0,
$$

holds. The iterates (16) contract in the space $E^{p, r}([0, T], \Omega)$ that is the completion of $C\left([0, T], L^{r}(\Omega)\right)$ for some $T$ sufficiently small, in the norm

$$
\|\vec{u}\|_{E^{p, r}} \equiv \sup \left\{\|\vec{u}\|_{L^{r} / L^{q}}: t \in[0, T]\right\} .
$$


At last, we show that Lemma 9 holds just as the condition (25) is satisfied. By the second equality of (33) and then the first one,

$$
\begin{aligned}
\frac{n}{2 s^{*}} & =\left(\frac{n}{2}\right)\left(1-\frac{1}{s}\right)=\left(\frac{n}{r}\right) \sigma=n \sigma\left(\frac{1}{p}+1-\frac{1}{q}\right) \\
& =\left(\frac{n}{p}\right) \sigma-\left(\frac{n}{q^{*}}\right) \sigma ;
\end{aligned}
$$

thus, we have $n / 2 s^{*}+\left(n / q^{*}\right) \sigma=(n / p) \sigma<1$, which means that the second inequality of (36) is seen to be equivalent to the condition

$$
\sigma n<p .
$$

Again, using (33), the first inequality of (36) becomes

$$
\begin{aligned}
\frac{n(2 \sigma+1)}{2 q^{*}} & =n\left(\sigma+\frac{1}{2}\right)\left(1-\frac{1}{q}\right) \\
& =n\left(\sigma+\frac{1}{2}\right)\left(\frac{1}{p}-\frac{1}{r}\right)<1,
\end{aligned}
$$

which implies that

$$
\frac{1}{p}-\frac{1}{(\sigma+1 / 2) n}<\frac{1}{r} .
$$

By a direct calculation and using the condition that $\sigma n<p$, we deduce

$$
r>(2 \sigma+1) p
$$

then, one sees that (42) is satisfied by choosing $r=(2 \sigma+$ 1) $p$; thus, condition (37) is met and the contraction mapping argument yields a unique solution $\vec{u}$ in $E^{p, r}([0, T], \Omega)$ for $r=$ $(2 \sigma+1) p$.

This solution is in $C\left([0, T], L^{p}(\Omega)\right) \cap C\left((0, T], L^{r}(\Omega)\right)$. Indeed, it is clear from the definition of the $E^{p, r}$-norm (38) that $E^{p, r}([0, T], \Omega) \subset C\left((0, T], L^{r}(\Omega)\right)$. To see that $\vec{u}$ is also in $C\left([0, T], L^{p}(\Omega)\right)$, one only needs to check the continuity of $\vec{u}$ at $t=0$. First, subtracting $\vec{u}_{0}$ from both sides of the mild formulation (15), a direct $L^{p}$ estimate gives

$$
\begin{aligned}
\left\|\vec{u}-\vec{u}_{0}\right\|_{L^{p}} \leq & \left\|N_{t} * \vec{u}_{0}-\vec{u}_{0}\right\|_{L^{p}} \\
& +\int_{0}^{t}\left\|N_{t-\tau}\right\|_{L^{1}}\left\|N_{\tau}\right\|_{L^{q}}^{2 \sigma+1}\|\vec{u}(\tau)\|_{L^{r} / L^{q}}^{2 \sigma+1} d \tau,
\end{aligned}
$$

where $r=(2 \sigma+1) p$ and $q$ are defined in (33). The strong $L^{p}$ continuity of the linear semigroup implies that the first term on the right side vanishes as $t$ tends to zero. The boundedness of $\vec{u}$ in $E^{p, r}$ and the $L^{1}$ bound (27) of $N_{t}$ imply that the second term on the right side will also vanish as $t$ tends to zero whenever the singularity of $\left\|N_{\tau}\right\|_{L^{q}}^{2 \sigma+1}$ at $\tau=0$ is integrable. By (33), this will be the case if and only if

$$
\left(\frac{n}{2 q^{*}}\right)(2 \sigma+1)<1,
$$

which was already controlled in (36) by the assumption that $\sigma n<p$. Therefore, $\vec{u}$ is continuous at $t=0$ and hence is in $C\left([0, T], L^{p}(\Omega)\right)$. This is Lemma 9 .
Lemma 10 ( $L^{p}$-local strong solutions). If p satisfies

$$
1 \leq p, \quad \sigma n<p
$$

then for every $\rho>0$ there exists a time $T(\rho)>0$ such that for every initial data $\vec{u}_{0} \in L^{p}(\Omega)$ with $\left\|\vec{u}_{0}\right\|_{L^{p}} \leq \rho$ there exists a unique

$$
\begin{aligned}
\vec{u} \in & C\left([0, T], L^{p}(\Omega)\right) \\
& \cap C\left((0, T], C^{2}(\Omega)\right) \cap C^{1}((0, T], C(\Omega)),
\end{aligned}
$$

satisfying the initial value problem (1)-(4).

Proof. In order to verify that a mild solution in $E^{p, r}([0, T], \Omega)$ is a strong solution in $C\left((0, T), C^{2}(\Omega)\right) \cap C^{1}((0, T], C(\Omega))$, we follow from Lemma 4 upon showing it is in $C\left((0, T], L^{\infty}(\Omega)\right)$. Thus, if $p>(\sigma+1 / 2) n$ then every $r \in[(2 \sigma+1) p, \infty]$ satisfies these criteria, and in particular $\vec{u} \in C\left((0, T], L^{\infty}(\Omega)\right)$. If, on the other hand, $\sigma n<p \leq(\sigma+1 / 2) n$ then the solution is at least in $C\left((0, T], L^{p_{1}}(\Omega)\right)$ for $p_{1}=(2 \sigma+1) p$. Then, when $p_{1}=(2 \sigma+1) p \geq(\sigma+1 / 2) n$, a second application of the above argument then shows that $\vec{u} \in C\left((0, T], L^{\infty}(\Omega)\right)$. More generally, a simple argument shows that whenever

$$
p_{l}=(2 \sigma+1)^{l} p \geq\left(\sigma+\frac{1}{2}\right) n, \quad \text { for some } l=1,2,3, \ldots,
$$

after applying the above argument $l$ times, it follows that $\vec{u} \in$ $C\left((0, T], L^{p_{n}}(\Omega)\right)$, and one more application then shows that $\vec{u} \in C\left((0, T], L^{\infty}(\Omega)\right)$.

Combining the above argument with Lemmas 4 and 8 yields the desired result.

\section{Global Existence of Strong Solutions}

From Lemma 10 in the last section, one can find that it suffices to obtain global control of any $L^{p}$ norm where $p$ satisfies (46). Precisely, we have the following lemma.

Lemma 11. For $\sigma>0$ and assume that coefficients parameters satisfy the conditions in Theorem 1, there is

$$
\left\|u+g \varphi_{B}\right\|_{2}^{2}+\left\|\varphi_{B}\right\|_{2}^{2} \leq C_{3}\left(u_{0}, \varphi_{B 0}, T\right)
$$

And let $q>2$, provided

$$
\left|d_{r}\right| \leq \frac{2 d_{i} \sqrt{q-1}}{q-2} ;
$$

then

$$
\|u\|_{q}^{q},\left\|\varphi_{B}\right\|_{q}^{q},\left\|u+g \varphi_{B}\right\|_{q}^{q}+\left\|\varphi_{B}\right\|_{q}^{q} \leq C_{4}\left(u_{0}, \varphi_{B 0}, T\right) .
$$


Proof. A direct calculation and an application of Hölder's inequality yield

$$
\begin{gathered}
\frac{1}{2} \frac{d}{d t}\left\|u+g \varphi_{B}\right\|_{2}^{2} \\
\leq\left(\frac{a d_{i}}{|d|^{2}}-\frac{d_{i}}{|d|^{2} U}\right)\left\|u+g \varphi_{B}\right\|_{2}^{2} \\
+\operatorname{Re}\left[\int \frac{i g}{d U} \varphi_{B}\left(\overline{u+g \varphi_{B}}\right) d x\right] \\
\quad-\frac{b d_{i}}{|d|^{2}}\left(\int\left|u+g \varphi_{B}\right|^{2} d x\right)^{\sigma+1}, \\
\frac{1}{2} \frac{d}{d t}\left\|\varphi_{B}\right\|_{2}^{2}=\operatorname{Re}\left[\int \frac{i g}{U}\left(u+g \varphi_{B}\right)\left(\overline{\varphi_{B}}\right) d x\right] .
\end{gathered}
$$

For $\sigma>0$ this differential inequality shows

$$
\begin{aligned}
\frac{1}{2} \frac{d}{d t} & \left(\left\|u+g \varphi_{B}\right\|_{2}^{2}+\left\|\varphi_{B}\right\|_{2}^{2}\right) \\
\leq & \left(\frac{a d_{i}}{|d|^{2}}-\frac{d_{i}}{|d|^{2} U}+\frac{|g|}{2|d| U}+\frac{|g|}{2 U}\right)\left\|u+g \varphi_{B}\right\|_{2}^{2} \\
& +\left(\frac{|g|}{2|d| U}+\frac{|g|}{2 U}\right)\left\|\varphi_{B}\right\|_{2}^{2} .
\end{aligned}
$$

By Gronwall's inequality, we have

$$
\left\|u+g \varphi_{B}\right\|_{2}^{2}+\left\|\varphi_{B}\right\|_{2}^{2} \leq C\left(\left\|u_{0}+g \varphi_{B 0}\right\|_{2}^{2}+\left\|\varphi_{B 0}\right\|_{2}^{2}, T\right)
$$

which implies that the $L^{2}$ norm of $\vec{u}$ is uniformly bounded in time.

For $q>2$, multiplying (8) by $\left|u+g \varphi_{B}\right|^{q-2}\left(u+g \varphi_{B}\right)$ and taking the real part,

$$
\begin{aligned}
\frac{1}{q} \frac{d}{d t}\left\|u+g \varphi_{B}\right\|_{q}^{q} & \\
= & \operatorname{Re}\left[\left(\frac{c d_{i}}{4 m|d|^{2}}+i \frac{c d_{r}}{4 m|d|^{2}}\right)\right. \\
& \left.\times \int \Delta\left(u+g \varphi_{B}\right)\left|u+g \varphi_{B}\right|^{q-2}\left(\overline{u+g \varphi_{B}}\right) d x\right] \\
+ & \left(\frac{a d_{i}}{|d|^{2}}-\frac{d_{i}}{|d|^{2} U}\right)\left\|u+g \varphi_{B}\right\|_{q}^{q} \\
+ & \operatorname{Re}\left[\int \frac{i g}{d U} \varphi_{B}\left|u+g \varphi_{B}\right|^{q-2}\left(\overline{u+g \varphi_{B}}\right) d x\right] \\
- & \frac{b d_{i}}{|d|^{2}}\left\|u+g \varphi_{B}\right\|_{q+2 \sigma}^{q+2 \sigma} .
\end{aligned}
$$

Notice that

$$
\left|d_{r}\right| \leq \frac{2 d_{i} \sqrt{q-1}}{q-2}
$$

then,

$$
\begin{aligned}
& \operatorname{Re}\left[\left(\frac{c d_{i}}{4 m|d|^{2}}+i \frac{c d_{r}}{4 m|d|^{2}}\right)\right. \\
& \left.\times \int \Delta\left(u+g \varphi_{B}\right)\left|u+g \varphi_{B}\right|^{q-2}\left(\overline{u+g \varphi_{B}}\right) d x\right] \\
& \leq-\frac{c d_{i}}{4 m|d|^{2}} \cdot \frac{2 \sqrt{q-1}}{q-2} \\
& \times\left|\operatorname{Im}\left(\int \Delta\left(u+g \varphi_{B}\right)\left|u+g \varphi_{B}\right|^{q-2}\left(\overline{u+g \varphi_{B}}\right) d x\right)\right| \\
& -\frac{c d_{r}}{4 m|d|^{2}} \operatorname{Im}\left(\int \Delta\left(u+g \varphi_{B}\right)\left|u+g \varphi_{B}\right|^{q-2}\left(\overline{u+g \varphi_{B}}\right) d x\right) \\
& \leq-\frac{c d_{i}}{4 m|d|^{2}} \cdot \frac{2 \sqrt{q-1}}{q-2} \\
& \times\left|\operatorname{Im}\left(\int \Delta\left(u+g \varphi_{B}\right)\left|u+g \varphi_{B}\right|^{q-2}\left(\overline{u+g \varphi_{B}}\right) d x\right)\right| \\
& +\left|\frac{c d_{r}}{4 m|d|^{2}} \operatorname{Im}\left(\int \Delta\left(u+g \varphi_{B}\right)\left|u+g \varphi_{B}\right|^{q-2}\left(\overline{u+g \varphi_{B}}\right) d x\right)\right| \\
& =\left(\left|\frac{c d_{r}}{4 m|d|^{2}}\right|-\frac{c d_{i}}{4 m|d|^{2}} \cdot \frac{2 \sqrt{q-1}}{q-2}\right) \\
& \times\left|\operatorname{Im}\left(\int \Delta\left(u+g \varphi_{B}\right)\left|u+g \varphi_{B}\right|^{q-2}\left(\overline{u+g \varphi_{B}}\right) d x\right)\right| \leq 0 .
\end{aligned}
$$

Noting that $b d_{i} \geq 0$, (55) becomes

$$
\begin{aligned}
\frac{d}{d t}\left\|u+g \varphi_{B}\right\|_{q}^{q} \leq & q\left(\frac{a d_{i}}{|d|^{2}}-\frac{d_{i}}{|d|^{2} U}+\frac{|g|}{|d| U}\right)\left\|u+g \varphi_{B}\right\|_{q}^{q} \\
& +\frac{q|g|}{|d| U}\left\|\varphi_{B}\right\|_{q}^{q} .
\end{aligned}
$$

Using Fourier transform, (9) can be reformed into

$$
\begin{aligned}
\varphi_{B}(x, t)= & e^{i H t} \varphi_{B 0}+\frac{i g}{U} \int_{0}^{t} e^{i H(t-\tau)}\left(u+g \varphi_{B}\right) d \tau \\
& -i\left(\frac{g^{2}}{U}+2 \nu-2 \mu\right) \int_{0}^{t} e^{i H(t-\tau)} \varphi_{B} d \tau,
\end{aligned}
$$

where $e^{i H t}=F^{-1}\left(e^{-(i / 4 m) \lambda^{2} t} F\right)$.

Let $q>2$ and $1 / q+1 / q^{\prime}=1$; then, from the properties of Besov space $[21,22]$, for $k>q^{\prime}$,

$$
\begin{aligned}
\left\|e^{i H t} \varphi_{B 0}\right\|_{B_{q^{\prime}, 2}^{0}} & \leq C\left\|e^{i H t} \varphi_{B 0}\right\|_{q^{\prime}, 2} \leq C\left\|e^{i H t} \varphi_{B 0}\right\|_{k, 2} \\
& =C\left\|\varphi_{B 0}\right\|_{k, 2} \leq C .
\end{aligned}
$$


The integral equation (59) gives [23]

$$
\begin{aligned}
\left\|\varphi_{B}(x, t)\right\|_{B_{q, 2}^{0}}^{q} \leq & C+C \int_{0}^{t}(t-s)^{\gamma}\left\|u+g \varphi_{B}\right\|_{B_{q^{\prime}, 2}^{0}}^{q} d \tau \\
& +C \int_{0}^{t}(t-s)^{\gamma}\left\|\varphi_{B}\right\|_{B_{q^{\prime}, 2}^{0}}^{q} d \tau
\end{aligned}
$$

where $0>\gamma=(q n / 2)\left(1-2 / q^{\prime}\right)>-q n / 2$. Thus,

$$
\left\|\varphi_{B}(x, t)\right\|_{q}^{q} \leq C+C \int_{0}^{t}\left\|u+g \varphi_{B}\right\|_{q^{\prime}}^{q^{\prime}} d \tau+C \int_{0}^{t}\left\|\varphi_{B}\right\|_{q^{\prime}}^{q^{\prime}} d \tau .
$$

Noting that $q^{\prime} \leq q$, then, by Hölder's and Young's inequalities, we have

$$
\left\|\varphi_{B}(x, t)\right\|_{q}^{q} \leq C+C \int_{0}^{t}\left\|u+g \varphi_{B}\right\|_{q}^{q} d \tau+C \int_{0}^{t}\left\|\varphi_{B}\right\|_{q}^{q} d \tau .
$$

And (58) implies

$$
\begin{aligned}
\left\|u+g \varphi_{B}\right\|_{q}^{q} \leq & q\left(\frac{a d_{i}}{|d|^{2}}-\frac{d_{i}}{|d|^{2} U}+\frac{|g|}{|d| U}\right) \int_{0}^{t}\left\|u+g \varphi_{B}\right\|_{q}^{q} d \tau \\
& +\frac{q|g|}{|d| U} \int_{0}^{t}\left\|\varphi_{B}\right\|_{q}^{q} d \tau+\left\|u_{0}+g \varphi_{B 0}\right\|_{q}^{q} .
\end{aligned}
$$

The former two inequalities mean that

$$
\begin{aligned}
\left\|u+g \varphi_{B}\right\|_{q}^{q}+\left\|\varphi_{B}(x, t)\right\|_{q}^{q} \\
\quad \leq C_{5}+C_{6} \int_{0}^{t}\left(\left\|u+g \varphi_{B}\right\|_{q}^{q}+\left\|\varphi_{B}\right\|_{q}^{q}\right) d \tau .
\end{aligned}
$$

By Gronwall's inequality, we obtain

$$
\left\|u+g \varphi_{B}\right\|_{q}^{q}+\left\|\varphi_{B}(x, t)\right\|_{q}^{q} \leq C_{4}\left(u_{0}, \varphi_{B 0}, T\right) .
$$

This completes the proof of Lemma 11.

Proof of Theorem 1. From Lemma 11, we can see that the $L^{2}$ norm of $\vec{u}$ is uniformly bounded in time. Then whenever

$$
\frac{1}{2} \sigma n-1<0
$$

Lemma 10 then implies that the problem (1)-(4) has global strong solutions.

When (67) is violated, one must control more than the $L^{2}$ norm. Thus when in addition to (50) one also has

$$
\sigma n<q,
$$

Lemma 10 then implies that the problem (1)-(4) has global strong solutions. We can find a $q>2$ satisfying both (50) and (68) provided, which means that

$$
\sigma n<\frac{2|d|}{|d|-d_{i}} .
$$

Thus, by Lemma 10, we get the global strong solutions for (1)-(4) with the $C^{2}$ initial conditions. This completes the Proof of Theorem 1.
Proof of Theorem 2. An alternation to directly controlling the $L^{p}$ norm is to rather first directly control the $H^{1}$ norm and then control $L^{p}$ through Sobolev estimate. As the $L^{2}$ norm is already controlled by (49), it suffices to control $L^{2}$ norm of $\nabla \vec{u}$. Assuming that $\sigma \geq 1 / 2$ and utilizing the decomposition identity

$$
\begin{aligned}
\mid u+ & \left.g \varphi_{B}\right|^{2}\left|\nabla\left(u+g \varphi_{B}\right)\right|^{2} \\
= & \frac{1}{4}|\nabla| u+\left.g \varphi_{B}\right|^{2} \mid \\
& +\frac{1}{4}\left|\left(\overline{u+g \varphi_{B}}\right) \nabla\left(u+g \varphi_{B}\right)-\left(u+g \varphi_{B}\right) \nabla\left(\overline{u+g \varphi_{B}}\right)\right|^{2},
\end{aligned}
$$

then a direct calculation shows that

$$
\begin{aligned}
\frac{1}{2} \frac{d}{d t}\left\|\nabla\left(u+g \varphi_{B}\right)\right\|^{2} & \\
= & -\frac{c d_{i}}{4 m|d|^{2}}\left\|\Delta\left(u+g \varphi_{B}\right)\right\|^{2} \\
& +\frac{d_{i}(a U-1)}{|d|^{2} U}\left\|\nabla\left(u+g \varphi_{B}\right)\right\|^{2} \\
& +\operatorname{Re}\left(\frac{i g}{d U} \int \nabla \varphi_{B} \nabla\left(\overline{u+g \varphi_{B}}\right) d x\right) \\
& +\operatorname{Re}\left[\left(\frac{b d_{i}}{|d|^{2}}+i \frac{b d_{r}}{|d|^{2}}\right)\right. \\
\quad & \left.\times \int \Delta\left(\overline{u+g \varphi_{B}}\right)\left(\left|u+g \varphi_{B}\right|^{2 \sigma}\left(u+g \varphi_{B}\right)\right) d x\right] .
\end{aligned}
$$

Similarly, as the estimate of (57),

$$
\begin{aligned}
& \operatorname{Re}\left[\left(\frac{b d_{i}}{|d|^{2}}+i \frac{b d_{r}}{|d|^{2}}\right)\right. \\
& \left.\quad \times \int \Delta\left(\overline{u+g \varphi_{B}}\right)\left(\left|u+g \varphi_{B}\right|^{2 \sigma}\left(u+g \varphi_{B}\right)\right) d x\right] \\
& \leq\left(\frac{b\left|d_{r}\right|}{|d|^{2}}-\frac{b d_{i}}{|d|^{2}} \cdot \frac{\sqrt{2 \sigma+1}}{\sigma}\right) \\
& \quad \times\left|\operatorname{Im}\left(\int \Delta\left(\overline{u+g \varphi_{B}}\right)\left(\left|u+g \varphi_{B}\right|^{2 \sigma}\left(u+g \varphi_{B}\right)\right) d x\right)\right| .
\end{aligned}
$$

Provided that

$$
\left|d_{r}\right| \leq \frac{d_{i} \sqrt{2 \sigma+1}}{\sigma}
$$


the last term of (71) will be nonpositive. And then it becomes

$$
\begin{aligned}
\frac{1}{2} \frac{d}{d t}\left\|\nabla\left(u+g \varphi_{B}\right)\right\|^{2} \\
\leq\left(\frac{d_{i}(a U-1)}{|d|^{2} U}+\frac{|g|}{2|d| U}\right)\left\|\nabla\left(u+g \varphi_{B}\right)\right\|^{2} \\
\quad+\frac{|g|}{2|d| U}\left\|\nabla \varphi_{B}\right\|^{2} .
\end{aligned}
$$

Now, multiplying (9) with $\Delta \varphi_{B}$ and taking the real part,

$$
\frac{1}{2} \frac{d}{d t}\left\|\nabla \varphi_{B}\right\|^{2} \leq \frac{|g|}{2 U}\left\|\nabla\left(u+g \varphi_{B}\right)\right\|^{2}+\frac{|g|}{2 U}\left\|\nabla \varphi_{B}\right\|^{2} .
$$

Combining (74) and (75) gives

$$
\begin{aligned}
\frac{1}{2} \frac{d}{d t} & \left(\left\|\nabla\left(u+g \varphi_{B}\right)\right\|^{2}+\left\|\nabla \varphi_{B}\right\|^{2}\right) \\
\leq & \left(\frac{d_{i}(a U-1)}{|d|^{2} U}+\frac{|g|}{2|d| U}+\frac{|g|}{2 U}\right) \\
& \times\left(\left\|\nabla\left(u+g \varphi_{B}\right)\right\|^{2}+\left\|\nabla \varphi_{B}\right\|^{2}\right) .
\end{aligned}
$$

Via Gronwall's inequality, we gain

$$
\begin{aligned}
\| \nabla(u & \left.+g \varphi_{B}\right)\left\|^{2}+\right\| \nabla \varphi_{B} \|^{2} \\
& \leq C\left(\left\|\nabla\left(u_{0}+g \varphi_{B 0}\right)\right\|_{2}^{2},\left\|\nabla \varphi_{B 0}\right\|_{2}^{2}, T\right) .
\end{aligned}
$$

This differential inequality shows that the $L^{2}$ norm of $\nabla \vec{u}$ for $\vec{u}=\left(\begin{array}{c}u+g \varphi_{B} \\ \varphi_{B}\end{array}\right)$ is uniformly bounded in time. This then gives a priori control of the $H^{1}$ norm if condition (73) is satisfied.

Control of the $H^{1}$ norm yields, by a Sobolev inequality, the $L^{p}$ norm for every $p$ satisfying

$$
1 \leq p< \begin{cases}\infty, & \text { for } n=1,2, \\ \frac{2 n}{n-2}, & \text { for } n \geq 3 .\end{cases}
$$

When, in addition to (73) and (78), one also has

$$
\sigma n<p \text {. }
$$

Lemma 10 implies that the problem (1)-(4) has global strong solutions. Given that $d_{i}, d_{r}$, and $\sigma$ satisfy (73), we can find a $p$ satisfying both (78) and (79), provided that

$$
n<2+\frac{2}{\sigma}
$$

This encompasses all subcritical and critical nonlinearities in every spatial dimension $(\sigma n \leq 2)$, the supercritical cubic nonlinearity $(\sigma=1)$ in dimension $n=3$, and all nonlinearities in dimensions $n \leq 2$.

Equations (65) and (77) may be combined to gain control on a set that is actually much larger than the union of the strips (50) and (73) about the axes of the $\left(c d_{r} / 4 m|d|^{2}, b d_{r} /|d|^{2}\right)$-plane. This is reflected in the condition of (7) in Theorem 2.
We will produce a bounds on a functional of the form

$$
\begin{aligned}
F=\int\left(\left|\nabla\left(u+g \varphi_{B}\right)\right|^{2}+\left|\nabla \varphi_{B}\right|^{2}\right) \\
\\
\quad+\frac{\beta^{2}}{\sigma+1}\left(\left|u+g \varphi_{B}\right|^{2 \sigma+2}+\left|\varphi_{B}\right|^{2 \sigma+2}\right) d x,
\end{aligned}
$$

where the parameter $\beta>0$ is to be chosen later.

From (70), we can obtain the identities

$$
\begin{gathered}
\int\left|\Delta\left(u+g \varphi_{B}\right) \mp \frac{2 \beta \sqrt{m b}}{\sqrt{c}}\right| u+\left.\left.g \varphi_{B}\right|^{2 \sigma}\left(u+g \varphi_{B}\right)\right|^{2} d x \\
=\int\left|\Delta\left(u+g \varphi_{B}\right)\right|^{2}+\frac{4 m b \beta^{2}}{c}\left|u+g \varphi_{B}\right|^{4 \sigma+2} d x \\
\pm \frac{1}{2} \int\left|u+g \varphi_{B}\right|^{2 \sigma-2} \\
\times\left[\frac{2 \beta \sqrt{m b}}{\sqrt{c}}(1+2 \sigma)|\nabla| u+\left.\left.g \varphi_{B}\right|^{2}\right|^{2}\right. \\
+\frac{2 \beta \sqrt{m b}}{\sqrt{c}} \mid\left(\overline{u+g \varphi_{B}}\right) \nabla\left(u+g \varphi_{B}\right) \\
\left.-\left.\left(u+g \varphi_{B}\right) \nabla\left(\overline{u+g \varphi_{B}}\right)\right|^{2}\right] d x .
\end{gathered}
$$

Taking a linear combination of (55), (62), (74), and (75), while using (82), and noting that $c d_{i}>0$ give

$$
\begin{aligned}
& \frac{1}{2} \frac{d F}{d t} \\
& \leq\left(\frac{d_{i}(a U-1)}{|d|^{2} U}+\frac{|g|}{2|d| U}+\frac{|g|}{2 U}\right) \\
& \quad \times \int\left|\nabla\left(u+g \varphi_{B}\right)\right|^{2}+\left|\nabla \varphi_{B}\right|^{2} d x \\
& \quad+\beta^{2}\left(\frac{d_{i}(a U-1)}{|d|^{2} U}+\frac{|g|}{|d| U}+C\right) \\
& \quad \times \int\left|u+g \varphi_{B}\right|^{2 \sigma+2}+\left|\varphi_{B}\right|^{2 \sigma+2} d x \\
& \quad-\frac{1}{2}(1-\delta) \frac{c d_{i}}{4 m|d|^{2}} \\
& \quad \times \int\left|\Delta\left(u+g \varphi_{B}\right)-\frac{2 \beta \sqrt{m b}}{\sqrt{c}}\right| u+\left.\left.g \varphi_{B}\right|^{2 \sigma}\left(u+g \varphi_{B}\right)\right|^{2} d x \\
& \quad \times \int\left|\Delta\left(u+g \varphi_{B}\right)+\frac{2 \beta \sqrt{m b}}{\sqrt{c}}\right| u+\left.\left.g \varphi_{B}\right|^{2 \sigma}\left(u+g \varphi_{B}\right)\right|^{2} d x \\
& \quad-\frac{1}{2}(1+\delta) \frac{c d_{i}}{4 m|d|^{2}} \\
& \quad+\left.d\right|^{2}
\end{aligned}
$$


Advances in Mathematical Physics

9

$$
\begin{gathered}
\times \int\left|u+g \varphi_{B}\right|^{2 \sigma-2}\left[\frac{2 \beta \sqrt{m b}}{\sqrt{c}}(1+2 \sigma)|\nabla| u+\left.\left.g \varphi_{B}\right|^{2}\right|^{2}\right. \\
+\frac{2 \beta \sqrt{m b}}{\sqrt{c}} \\
\times \mid\left(\overline{u+g \varphi_{B}}\right) \nabla\left(u+g \varphi_{B}\right) \\
\left.-\left.\left(u+g \varphi_{B}\right) \nabla\left(\overline{u+g \varphi_{B}}\right)\right|^{2}\right] d x \\
+\operatorname{Re}\left[\left(\frac{b d_{i}}{\left.|d|^{2}+i \frac{b d_{r}}{|d|^{2}}\right)}\right.\right. \\
\left.\times \int \Delta\left(\overline{u+g \varphi_{B}}\right)\left(\left|u+g \varphi_{B}\right|^{2 \sigma}\left(u+g \varphi_{B}\right)\right) d x\right] \\
+\operatorname{Re}\left[\beta^{2}\left(\frac{c d_{i}}{4 m|d|^{2}}+i \frac{c d_{r}}{4 m|d|^{2}}\right)\right. \\
\left.\quad \times \int \Delta\left(u+g \varphi_{B}\right)\left(\left|u+g \varphi_{B}\right|^{2 \sigma}\left(\overline{u+g \varphi_{B}}\right)\right) d x\right],
\end{gathered}
$$

$$
\begin{aligned}
& \operatorname{Re}\left[\left(\frac{b d_{i}}{|d|^{2}}+i \frac{b d_{r}}{|d|^{2}}\right)\right. \\
& \left.\times \int \Delta\left(\overline{u+g \varphi_{B}}\right)\left(\left|u+g \varphi_{B}\right|^{2 \sigma}\left(u+g \varphi_{B}\right)\right) d x\right] \\
& =-\frac{b}{4|d|^{2}} \\
& \times \int\left|u+g \varphi_{B}\right|^{2 \sigma-2} \\
& \times\left[(2 \sigma+1) d_{i}|\nabla| u+\left.\left.g \varphi_{B}\right|^{2}\right|^{2}\right. \\
& +2 \sigma d_{r} \nabla\left|u+g \varphi_{B}\right|^{2} \\
& \cdot i\left(\left(u+g \varphi_{B}\right) \nabla\left(\overline{u+g \varphi_{B}}\right)\right. \\
& \left.-\left(\overline{u+g \varphi_{B}}\right) \nabla\left(u+g \varphi_{B}\right)\right) \\
& +d_{i} \mid\left(u+g \varphi_{B}\right) \nabla\left(\overline{u+g \varphi_{B}}\right) \\
& \left.-\left.\left(\overline{u+g \varphi_{B}}\right) \nabla\left(u+g \varphi_{B}\right)\right|^{2}\right] d x, \\
& \operatorname{Re}\left[\beta^{2}\left(\frac{c d_{i}}{4 m|d|^{2}}+i \frac{c d_{r}}{4 m|d|^{2}}\right)\right. \\
& \left.\times \int \Delta\left(u+g \varphi_{B}\right)\left(\left|u+g \varphi_{B}\right|^{2 \sigma}\left(\overline{u+g \varphi_{B}}\right)\right) d x\right] \\
& \frac{1}{2} \frac{d F}{d t} \\
& \leq\left(\frac{d_{i}(a U-1)}{|d|^{2} U}+\frac{|g|}{2|d| U}+\frac{|g|}{U}\right) \\
& \times \int\left|\nabla\left(u+g \varphi_{B}\right)\right|^{2}+\left|\nabla \varphi_{B}\right|^{2} d x \\
& +\beta^{2}\left(\frac{d_{i}(a U-1)}{|d|^{2} U}+\frac{|g|}{|d| U}+C\right) \\
& \times \int\left|u+g \varphi_{B}\right|^{2 \sigma+2}+\left|\varphi_{B}\right|^{2 \sigma+2} d x-\frac{1}{2}(1-\delta) \frac{c d_{i}}{4 m|d|^{2}} \\
& \times \int\left|\Delta\left(u+g \varphi_{B}\right)-\frac{2 \beta \sqrt{m b}}{\sqrt{c}}\right| u+\left.\left.g \varphi_{B}\right|^{2 \sigma}\left(u+g \varphi_{B}\right)\right|^{2} d x \\
& -\frac{1}{2}(1+\delta) \frac{c d_{i}}{4 m|d|^{2}} \\
& \times \int\left|\Delta\left(u+g \varphi_{B}\right)+\frac{2 \beta \sqrt{m b}}{\sqrt{c}}\right| u+\left.\left.g \varphi_{B}\right|^{2 \sigma}\left(u+g \varphi_{B}\right)\right|^{2} d x \\
& -\frac{1}{4|d|^{2}} \int\left|u+g \varphi_{B}\right|^{2 \sigma-2} \\
& \times\left\{(2 \sigma+1)\left(b d_{i}+\frac{c d_{i} \beta^{2}}{4 m}+\frac{\delta \beta d_{i} \sqrt{b c}}{\sqrt{m}}\right)|\nabla| u+\left.\left.g \varphi_{B}\right|^{2}\right|^{2}\right. \\
& +2 \sigma d_{r}\left(b-\frac{c \beta^{2}}{4 m}\right) \nabla\left|u+g \varphi_{B}\right|^{2} \\
& \cdot i\left[\left(u+g \varphi_{B}\right) \nabla\left(\overline{u+g \varphi_{B}}\right)-\left(\overline{u+g \varphi_{B}}\right) \nabla\left(u+g \varphi_{B}\right)\right] \\
& +\left(b d_{i}+\frac{c d_{i} \beta^{2}}{4 m}+\frac{\delta \beta d_{i} \sqrt{b c}}{\sqrt{m}}\right) \\
& \times \mid\left(u+g \varphi_{B}\right) \nabla\left(\overline{u+g \varphi_{B}}\right) \\
& \left.-\left.\left(\overline{u+g \varphi_{B}}\right) \nabla\left(u+g \varphi_{B}\right)\right|^{2}\right\} d x,
\end{aligned}
$$

$$
\begin{aligned}
& =-\frac{c \beta^{2}}{16 m|d|^{2}} \\
& \times \int\left|u+g \varphi_{B}\right|^{2 \sigma-2} \\
& \quad \times\left[(2 \sigma+1) d_{i}|\nabla| u+\left.\left.g \varphi_{B}\right|^{2}\right|^{2}\right. \\
& \quad+2 \sigma d_{r} \nabla\left|u+g \varphi_{B}\right|^{2} \\
& \quad \cdot i\left(\left(u+g \varphi_{B}\right) \nabla\left(\overline{u+g \varphi_{B}}\right)-\left(\overline{u+g \varphi_{B}}\right) \nabla\left(u+g \varphi_{B}\right)\right) \\
& \quad+d_{i} \mid\left(u+g \varphi_{B}\right) \nabla\left(\overline{u+g \varphi_{B}}\right) \\
& \left.\quad-\left.\left(\overline{u+g \varphi_{B}}\right) \nabla\left(u+g \varphi_{B}\right)\right|^{2}\right] d x .
\end{aligned}
$$

Then, from (84), we have 
for some $-1 \leq \delta \leq 1$ to be chosen. The last term above will be nonpositive provided the matrix

$$
\left(\begin{array}{cc}
(2 \sigma+1)\left(b d_{i}+\frac{c d_{i} \beta^{2}}{4 m}+\frac{\delta \beta d_{i} \sqrt{b c}}{\sqrt{m}}\right) & \sigma d_{r}\left(b-\frac{c \beta^{2}}{4 m}\right) \\
\sigma d_{r}\left(b-\frac{c \beta^{2}}{4 m}\right) & \left(b d_{i}+\frac{c d_{i} \beta^{2}}{4 m}+\frac{\delta \beta d_{i} \sqrt{b c}}{\sqrt{m}}\right)
\end{array}\right)
$$

is nonnegative defined, that is, whenever

$$
\sigma \leq 1 \times\left(\sqrt{1+\frac{d_{r}^{2}\left(b-c \beta^{2} / 4 m\right)^{2}}{d_{i}\left(b+\delta \beta \sqrt{b c} / \sqrt{m}+c \beta^{2} / 4 m\right)^{2}}}-1\right)^{-1} .
$$

In that case, neglecting the last three terms on the right of (85) gives

$$
\begin{aligned}
\frac{1}{2} \frac{d F}{d t} \leq & \left(\frac{d_{i}(a U-1)}{|d|^{2} U}+\frac{|g|}{2|d| U}+\frac{|g|}{U}\right) \\
& \times \int\left|\nabla\left(u+g \varphi_{B}\right)\right|^{2}+\left|\nabla \varphi_{B}\right|^{2} d x \\
& +\beta^{2}\left(\frac{d_{i}(a U-1)}{|d|^{2} U}+\frac{|g|}{|d| U}+C\right) \\
& \times \int\left|u+g \varphi_{B}\right|^{2 \sigma+2}+\left|\varphi_{B}\right|^{2 \sigma+2} d x .
\end{aligned}
$$

This differential inequality shows that $F$ is uniformly bounded in time when $-1 \leq \delta<1$ and is exponentially bounded in time when $\delta=1$. This then gives a priori control $F$ if $d_{i}, d_{r}, m, b$, and $c$ are such that (88) is satisfied.

Control of the $F$ obviously gives control of the $H^{1}$ norm of $\vec{u}$. Assume that (80) and (88) are satisfied. The following choices for $\beta$ and $\delta$ will maximize the upper bounds (88) on $\sigma$. If $c=0$, then for any $b$ and $d_{r}$ we may choose a $\beta$ large enough to satisfy (88) for any fixed value of $\delta$, say, $1 / 2$, and there is no restriction, where we have used (69). If $d_{r}=0$, the result is obvious. If $c d_{r} / 4 m|d|^{2} \neq 0$ and $4 m b / c \geq 0$, then choose $\beta=\sqrt{4 m b / c}$ and there is again no restriction on $\sigma$. If $c d_{r} / 4 m|d|^{2} \neq 0$ and $c d_{r} / 4 m|d|^{2}$ and $b /|d|^{2}$ have the opposite signs, then choose $\beta=-4 \mathrm{mb} / \mathrm{c}$ for any value of $\delta$. The maximum of the upper bounds (88) on $\sigma$ is then obtained by setting $\delta=1$ and yields (69).

\section{Conflict of Interests}

The authors declare that there is no conflict of interests regarding the publication of this paper.

\section{Acknowledgment}

This work is supported by National Natural Science Foundation of China (Grants nos. 11201415, 11271305); Program for New Century Excellent Talents in Fujian Province University (Grant no. JA14191).

\section{References}

[1] M. Machida and T. Koyama, "Time-dependent GinzburgLandau theory for atomic Fermi gases near the BCS-BEC crossover," Physical Review A, vol. 74, no. 3, Article ID 033603, 2006.

[2] M. Drechsler and W. Zwerger, "Crossover from BCSsuperconductivity to Bose-condensation," Annals of Physics, vol. 504, no. 1, pp. 15-23, 1992.

[3] C. A. R. Sá de Melo, M. Randeria, and J. R. Engelbrecht, "Crossover from BCS to Bose superconductivity: transition temperature and time-dependent Ginzburg-Landau theory," Physical Review Letters, vol. 71, no. 19, pp. 3202-3205, 1993.

[4] Y. He, Q. Chen, C. C. Chien, and K. Levin, "First- and secondsound-like modes at finite temperature in trapped Fermi gases from BCS to BEC," Physical Review A, vol. 76, no. 5, Article ID 051602, 2007.

[5] T. N. de Silva, "Breathing mode frequencies of a rotating Fermi gas in the BCS-BEC crossover region," Physical Review A, vol. 78, no. 2, Article ID 023623, 2008.

[6] Z. Q. Yu, K. Huang, and L. Yin, "Induced interaction in a Fermi gas with a BEC-BCS crossover," Physical Review A, vol. 79, no. 5, Article ID 053636, 2009.

[7] P. G. de Gennes, Superconductivity of Metals and Alloys, Westview Press, 1999.

[8] A. A. Abrikosov, Fundamentals of the Theory of Metals, NorthHolland, 1988.

[9] C. R. Doering, J. D. Gibbon, D. D. Holm, and B. Nicolaenko, "Low-dimensional behaviour in the complex Ginzburg-Landau equation," Nonlinearity, vol. 1, no. 2, pp. 279-309, 1988.

[10] C. R. Doering, J. D. Gibbon, and C. D. Levermore, "Weak and strong solutions of the complex Ginzburg-Landau equation," Physica D: Nonlinear Phenomena, vol. 71, no. 3, pp. 285-318, 1994.

[11] L. Sirovich and J. D. Rodriguez, "Coherent structures and chaos: a model problem," Physics Letters. A, vol. 120, no. 5, pp. 211-214, 1987.

[12] S. Chen and B. Guo, "Existence of the weak solution of coupled time-dependent Ginzburg-Landau equations," Journal of Mathematical Physics, vol. 51, no. 3, Article ID 033507, 2010.

[13] S. Chen and B. Guo, "Classical solutions of time-dependent Ginzburg-Landau theory for atomic Fermi gases near the BCSBEC crossover," Journal of Differential Equations, vol. 251, no. 6, pp. 1415-1427, 2011.

[14] P. Brenner and W. von Wahl, "Global classical solutions of nonlinear wave equations," Mathematische Zeitschrift, vol. 176, no. 1, pp. 87-121, 1981.

[15] M. Reed, Abstract Non-Linear Wave Equations, vol. 507 of Lecture Notes in Mathematics, Springer, Berlin, Germany, 1976.

[16] T. Peter and S. Huang, "Global smooth solutions of the complex Ginzburg-Landau equation and their dynamical properties," Discrete and Continuous Dynamical Systems, vol. 5, no. 4, pp. 825-848, 1999.

[17] N. Hayashi, "Classical solutions of nonlinear Schrödinger equations," Manuscripta Mathematica, vol. 55, no. 2, pp. 171-190, 1986.

[18] H. Pecher, " $L^{p}$-Abschätzungen und klassische Lösungen für nichtlineare Wellengleichungen I," Mathematische Zeitschrift, vol. 150, pp. 159-183, 1976.

[19] M. Tsutsumi and N. Hayashi, "Classical solutions of nonlinear Schrödinger equations in higher dimensions," Mathematische Zeitschrift, vol. 177, no. 2, pp. 217-234, 1981. 
[20] H. Pecher, " $L^{p}$-Abschätzungen und klassische Lösungen für nichtlineare Wellengleichungen II," Manuscripta Mathematica, vol. 20, pp. 227-244, 1977.

[21] J. Bergh and J. Löfström, Interpolation Spaces, Springer, New York, NY, USA, 1976.

[22] H. Triebel, Interpolation Theory, Function Spaces, Differential Operators, North Holland, Amsterdam, The Netherlands, 1978.

[23] H. Pecher and W. von Wahl, "Time dependent nonlinear Schrödinger equations," Manuscripta Mathematica, vol. 27, no. 2, pp. 125-157, 1979. 


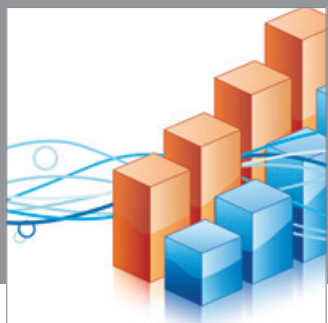

Advances in

Operations Research

mansans

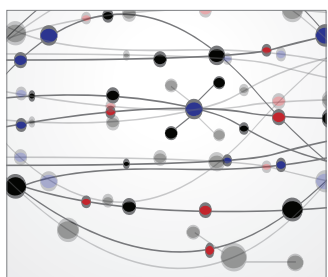

The Scientific World Journal
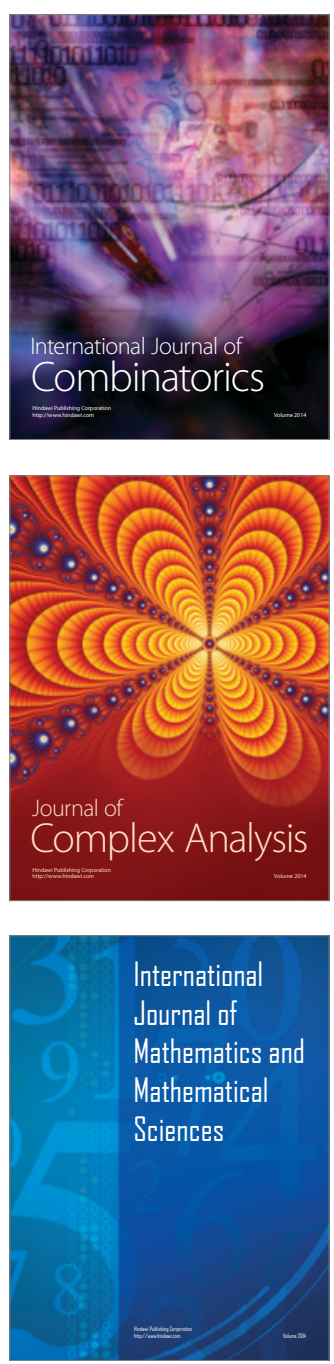
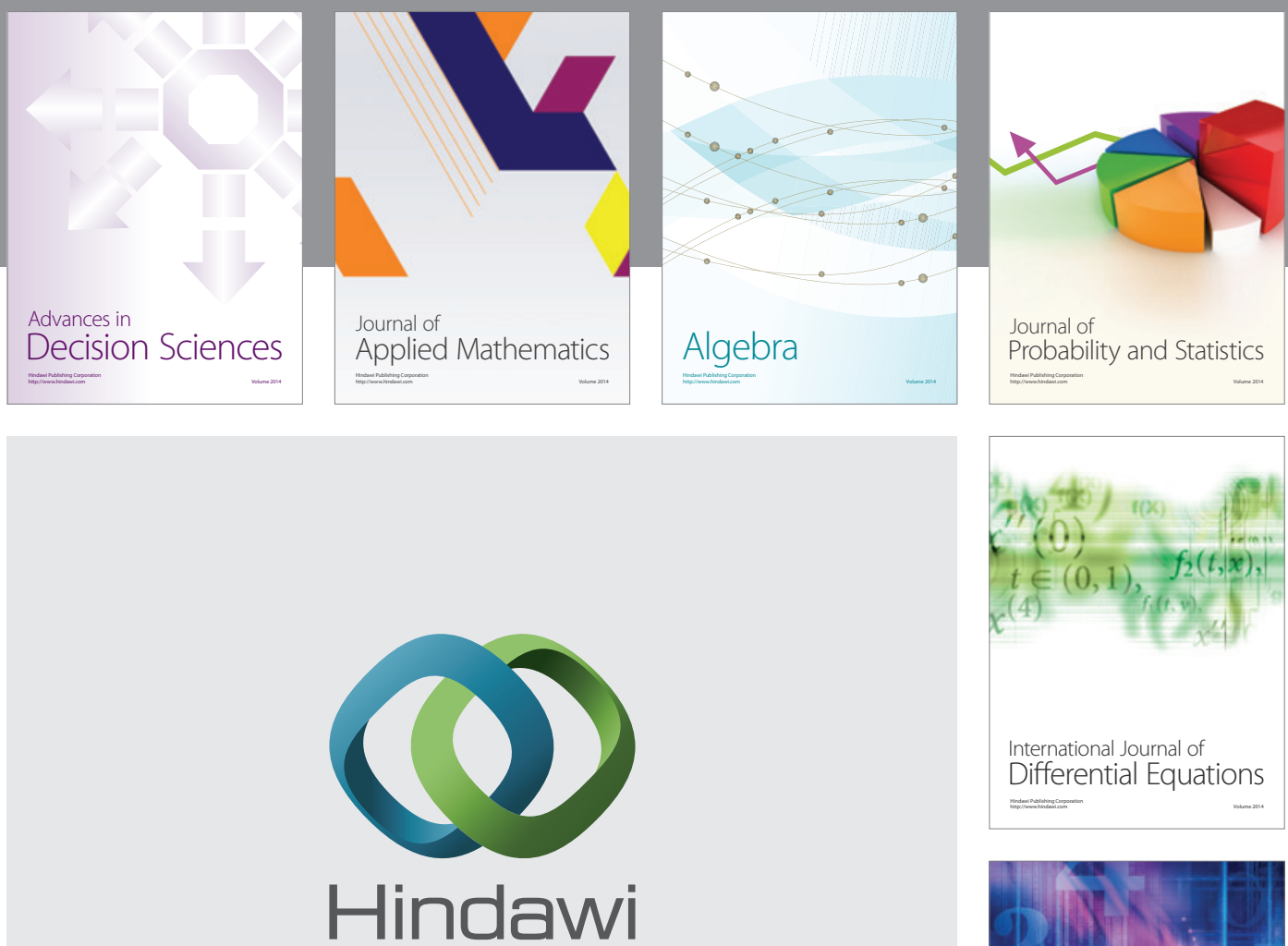

Submit your manuscripts at http://www.hindawi.com
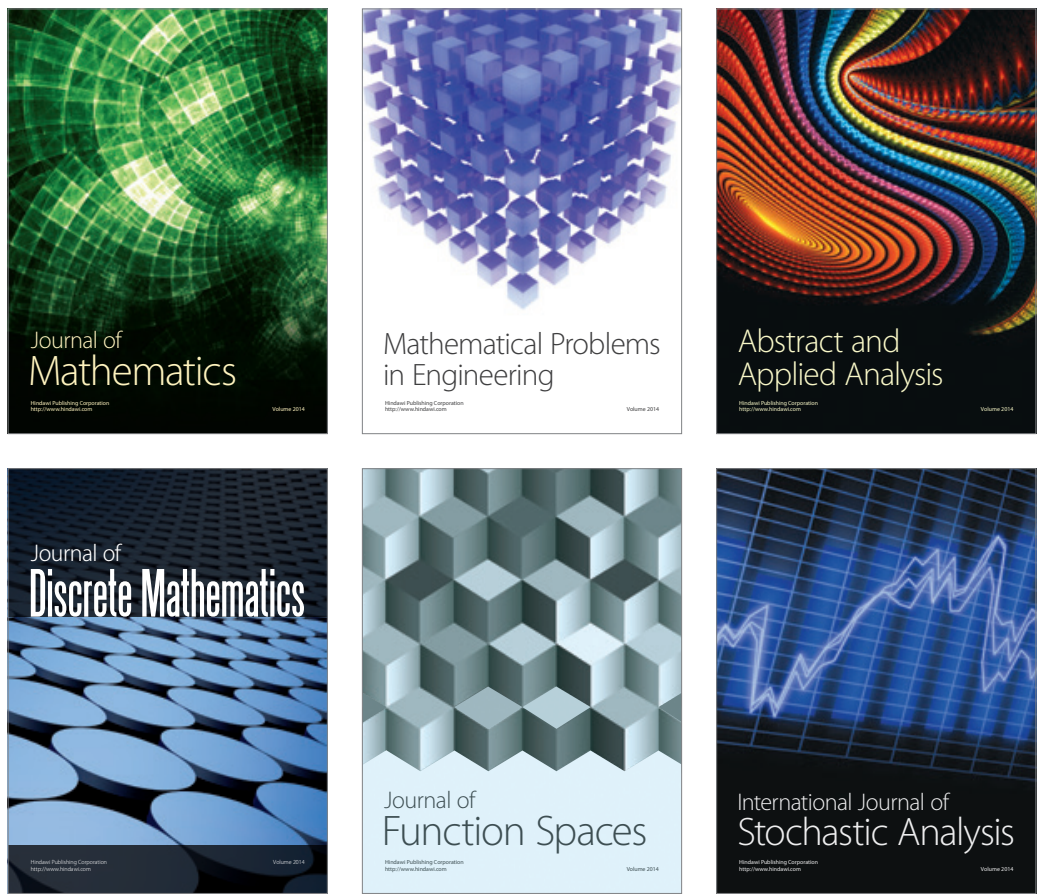

Journal of

Function Spaces

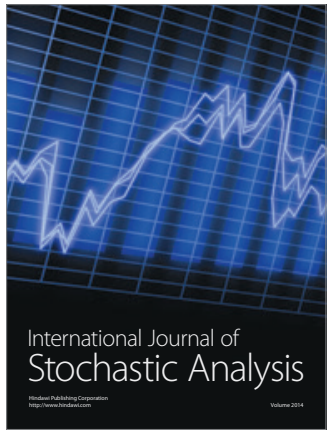

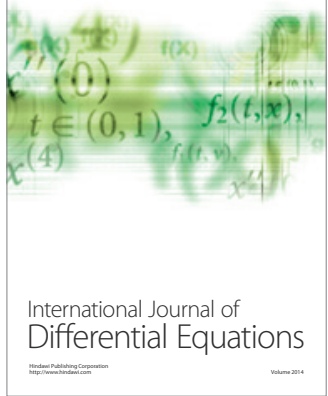
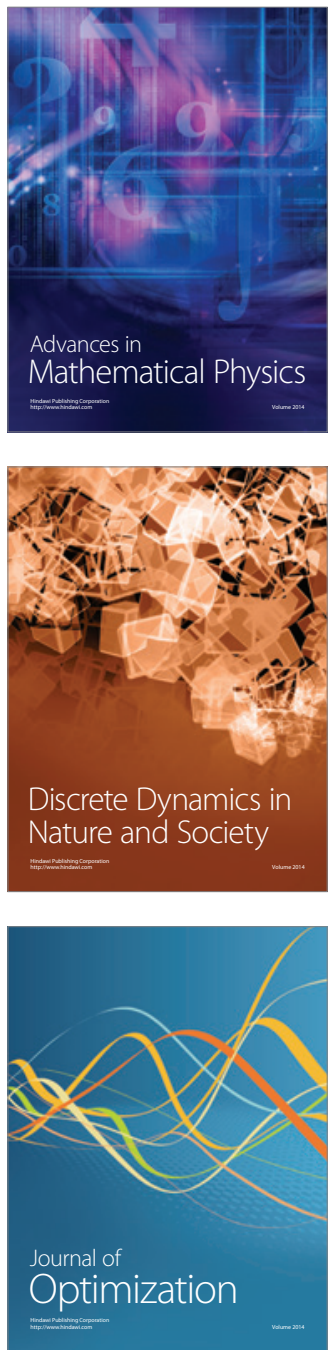